\title{
Soil Particle Movement and Shear Band Development during Plane Strain Compression
}

\author{
Junggeun Hwang ${ }^{1,}{ }^{*}$ and Hoe I. Ling ${ }^{1}$ \\ ${ }^{1}$ Columbia University, $116^{\text {th }}$ St. Broadway, New York, NY 10027, USA
}

\begin{abstract}
Most geotechnical structures failed by formation and development of shear bands in soils. Thus, shear deformation and shear bands development evaluation are necessary to understand shear failure mechanism. During shearing, deformation behaviour analysis for soil particles within entire soil specimen are evaluated to understand the soil behaviour and shear strength characteristics. In this paper, a series of plane strain compression tests using Nevada sand and Ottawa sand were conducted to identify the shear strain and shear failure mechanism. With the results of plane strain compression tests, image analyses using Particle Image Velocimetry (PIV) were carried out in order to measure the change in position of soil particles and shear bands development. Deformation vectors and contours were constructed to see the entire deformation mechanism in the soil specimen. During shearing, shear band was identified after peak stress and most visually distinctive at residual state. However, shear band started to develop invisibly immediately after starting loading and this invisible development was able to be observed by horizontal and vertical movement analyses of PIV. Soil particles moved actively in horizontal and vertical direction to generate shear band in the beginning of shearing. After development of shear band, soil particles moved along the shear band.
\end{abstract}

\section{Introduction}

Many researches which are about plane strain failure mechanism and shear bands have been carried out. However, they were limited to understand shear band formation by using grids on the membrane.

Photogrammetric analysis method was used with the assumption that grid on the membrane moves with soil particle movement [1]. Using grid analysis, shear band formation and thickness of shear bands according to the soil types were studied. [2] carried out researches about shear band formation using X-ray method. In this research, shear bands were able to be seen very clearly. Thickness, and direction variation according to the void inside were observed using X-ray method. [3] conducted research about shear bands formation according to the grain size of sand using Digital Image Correlation (DIC). In their researches, the local displacement of soil particles was observed using image analysis technique. However, it was hard to analyze since they used grid on the membrane. Researchers were able to observe overall behavior of soil particle and shear bands formation from outside through grid deformation. Therefore, it has been reported that shear bands can be observed right after peak stress and become clear during residual stress state. In this paper, formation process of vertical and horizontal shear bands separately during plane strain compression was observed.

\subsection{Test preparation}

Plane Strain Compression (PSC) tests were conducted using Ottawa sand and Nevada sand respectively. The properties of each sand are given below (Table1). Specimen was of size $160 \mathrm{~mm}(\mathrm{~L}) * 80 \mathrm{~mm}(\mathrm{~W}) * 200$ $\mathrm{mm}(\mathrm{H})$. It was enclosed with $0.3 \mathrm{~mm}$ of latex membrane and was saturated by freeze-thaw method. $93 \mathrm{kPa}$ of confining pressure was applied from inside of specimen by suction force. As a reference points, 12 circular points were attached to the plate for PIV analysis (Figure 1). During shearing, specimens were confined by steel and acrylic plate to prevent deformation of sigma 2 direction (Figure 2). Grease was used on both plates in order to minimize friction between membrane and confining plates. Axial strain rate was $0.9 \% / \mathrm{min}$. 4 load cells were used in order to obtain the applied load during shearing.

Table 1. Test materials

\begin{tabular}{|c|c|c|c|c|}
\hline Material & $\begin{array}{c}\mathbf{D}_{\text {50 }} \\
(\mathbf{m m})\end{array}$ & Gs & $\mathbf{e}_{\mathbf{m a x}}$ & $\mathbf{e}_{\mathbf{m i n}}$ \\
\hline $\begin{array}{c}\text { Nevada Sand } \\
{[4]}\end{array}$ & 0.14 & 2.65 & 0.748 & 0.51 \\
\hline $\begin{array}{c}\text { Ottawa Sand } \\
{[5]}\end{array}$ & 0.182 & 2.665 & 0.864 & 0.515 \\
\hline
\end{tabular}

\footnotetext{
* Corresponding author: hj2417@,columbia.edu.org
} 


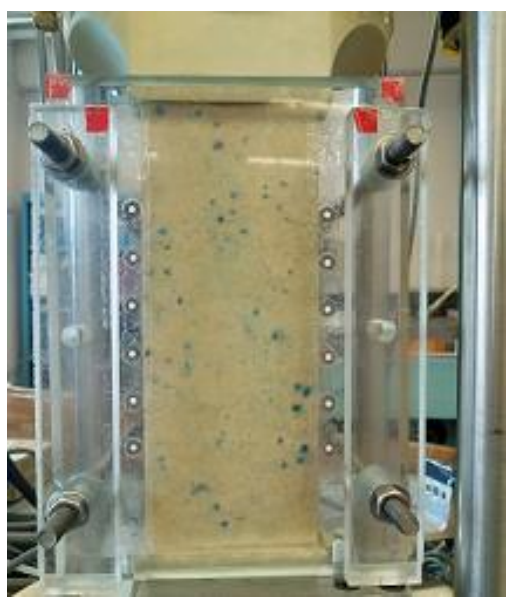

Fig. 1. Test Specimen

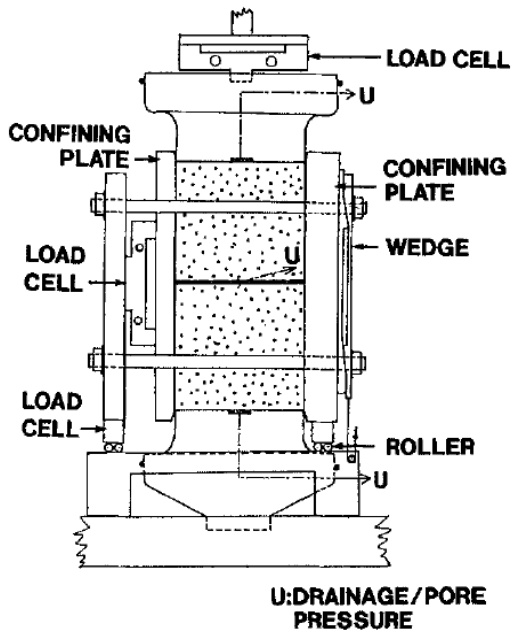

Fig. 2. Plane strain test apparatus [6]

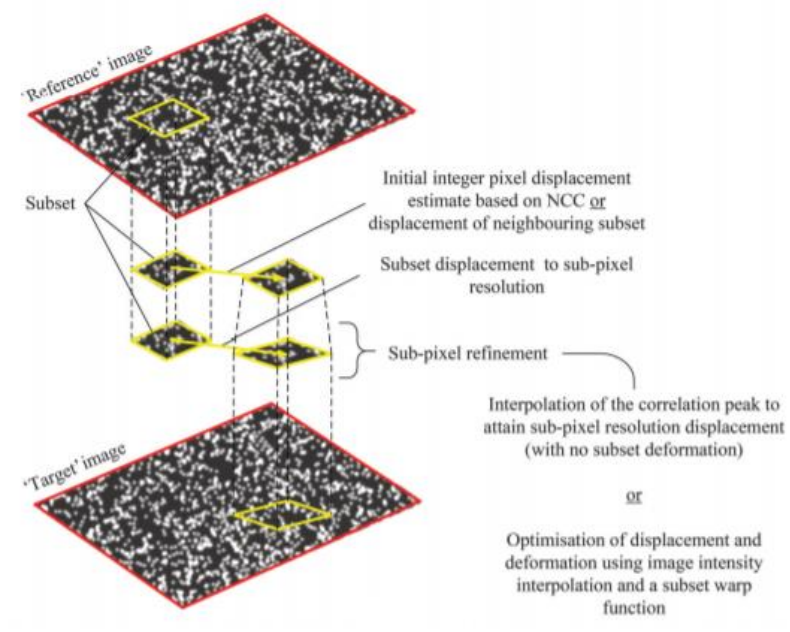

Fig. 3. GeoPIV_RG analysis concept [7]

\subsection{PIV analysis}

Particle image velocimetry (PIV) is able to track soil particles during shearing. In order to do the analysis, highresolution camera is required. However, it is difficult to make analysis for fine sand without colored reference points. For this test, blue colored flocking powder was used to chase the particle movement of pure sand. To prevent changes in soil properties due to flocking powder, flocking powder was scattered on only one side of specimen which was covered by acrylic plate. In this paper, GeoPIV-RG software was used for image analysis. GeoPIV-RG is based on the MATLAB image analysis module to observe geotechnical or structural displacements and deformations (Figure 3). During shearing, photos were taken at every 10 seconds interval for PIV analysis.

\section{Analysis of results}

So far, many researches about soil particle movement have been conducted using grid deformation analysis. In past decades, several researches were carried out using digital image correlation or X-ray method to see the movement inside. However, those methods were able to analyze entire movement of soil particle. Horizontal and vertical particle movement will be shown separately and entire particle movement will be shown in order to compare with previous researches. Soil movement under applied load was different according to the soil type.

\subsection{Vertical movement of soil particle}

In terms of vertical movement of soil particles, the soil particle started to form shear bands immediately after peak stress. Shear bands were able to be observed clearly at residual stress state. With the load applied, during shearing stages, bottom part of specimen started to be dense to create shear band. The blue triangular part at the bottom of specimen was a base of shear bands. However, shear bands were not able to be seen at the beginning (Figure 4). Thus, both directions of shear bands were able to be expected through this triangular area. During residual stress state, soil particle started to move to opposite direction to make another shear band. To make another direction of shear band, one more triangular area was developed at the top of specimen and become denser. However, there was no huge difference of vertical movement of soil particle according to the soil type in terms of vertical displacement.

\subsection{Horizontal movement of soil particle}

Soil particle started to move immediately after load applying. As shown below, there was big difference of soil particle movement according to the soil type. At the peak stress, both directions of shear bands were able to be observed and one direction of shear band started to be developed first. The first shear bands were able to be seen during shearing (Figure 5). This horizontal displacement showed that shear failure mode can be expected in advance before shear failure based on the horizontal movement of soil particle. At $2 \%$ of strain rate, another direction of shear band was already able to be observed unlike vertical movement of soil particle. 


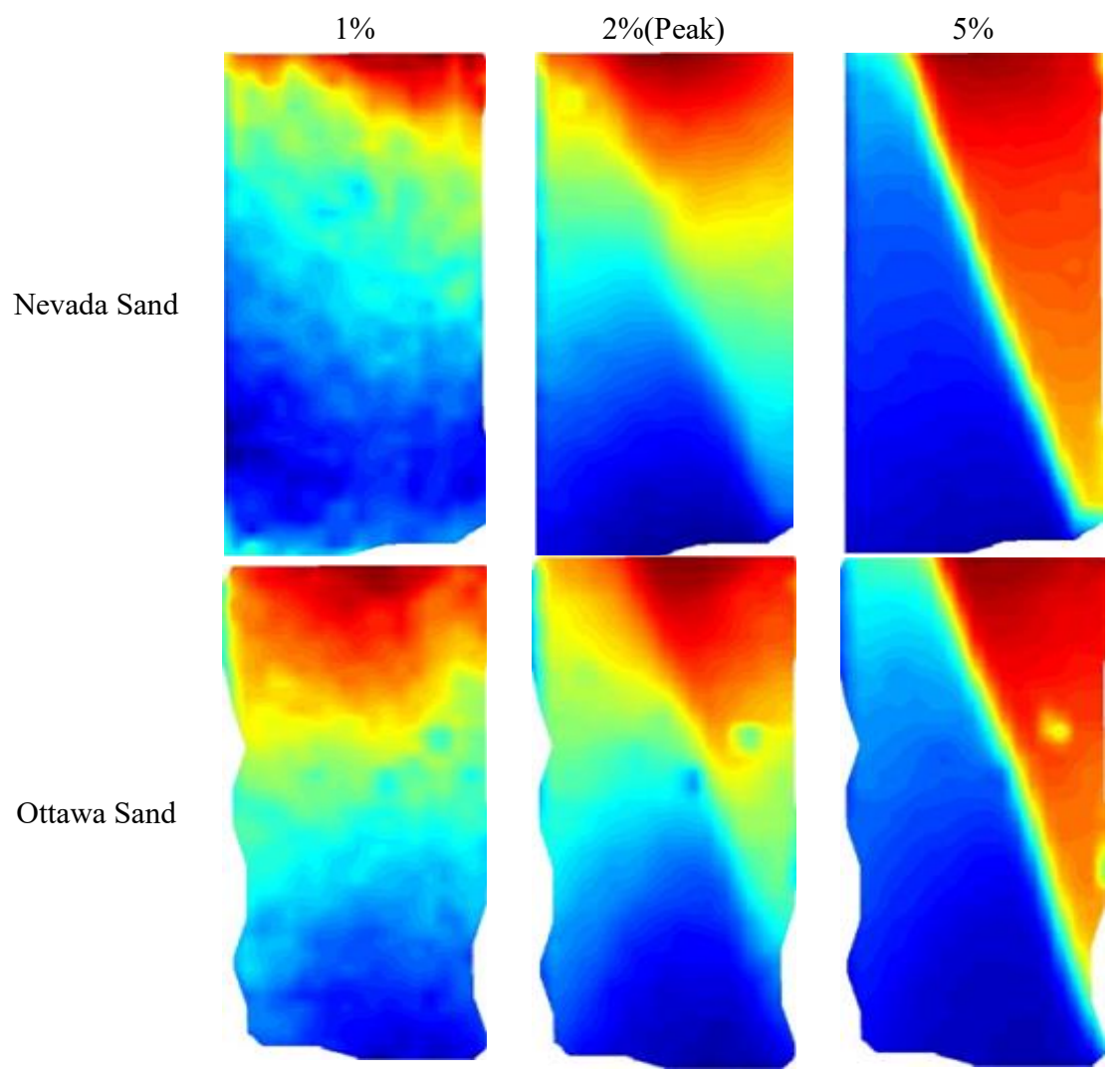

Fig. 4. Vertical shear band analysis using PIV
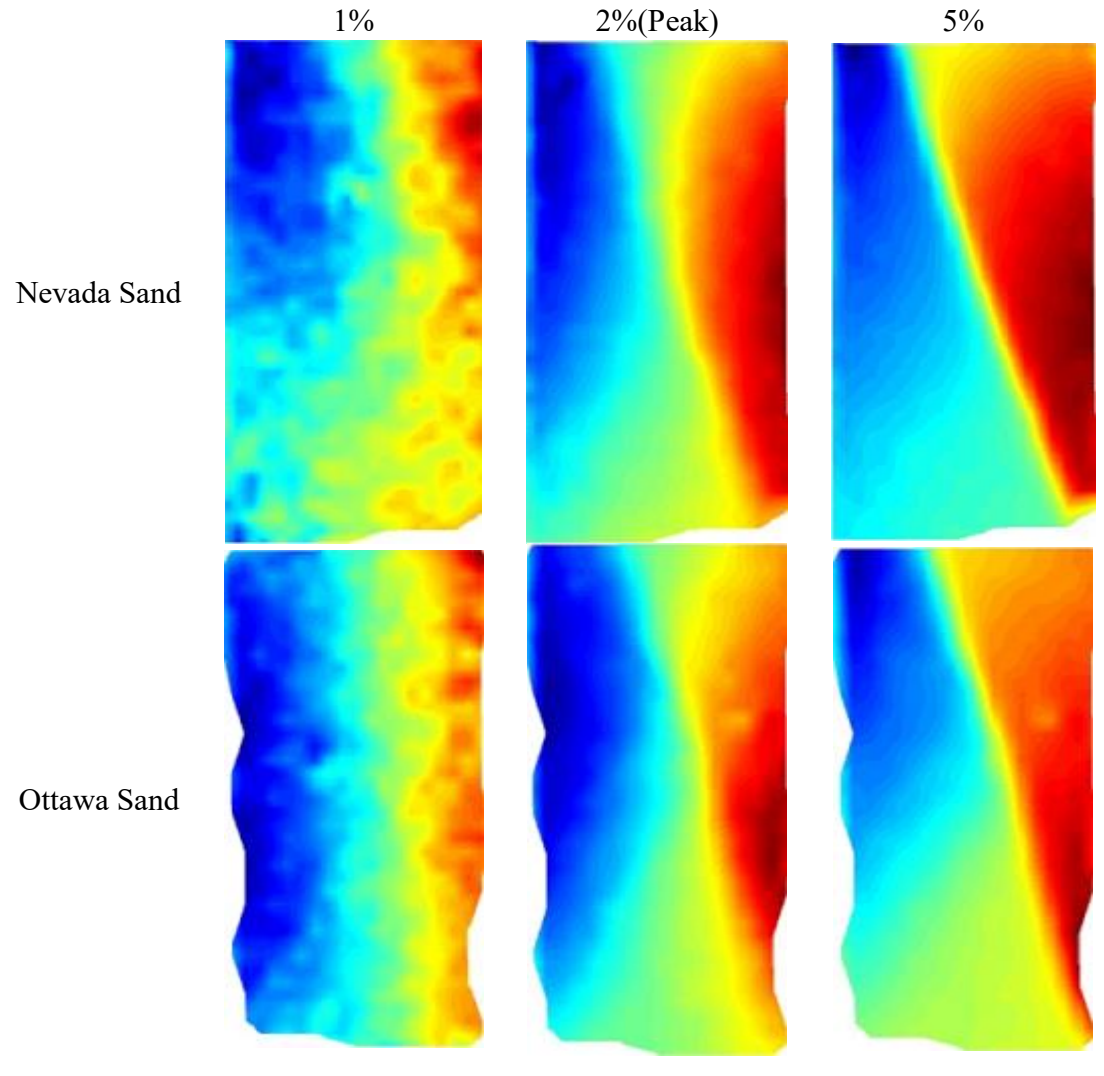

Fig. 5. Horizontal shear band analysis using PIV 


\subsection{Particle movement at residual stress state inside of shear bands}

So far, it has been known that soil particles which are in the shear bands moved along the shear surface. However, the movement of soil particles is different according to their location. At residual stress state, particles at the bottom of shear bands moved along the shear surface but the particles at the top of shear bands moved in opposite direction of triangle as mention above. Particle movement in the opposite direction is going to make another direction of shear band (Figure 6).
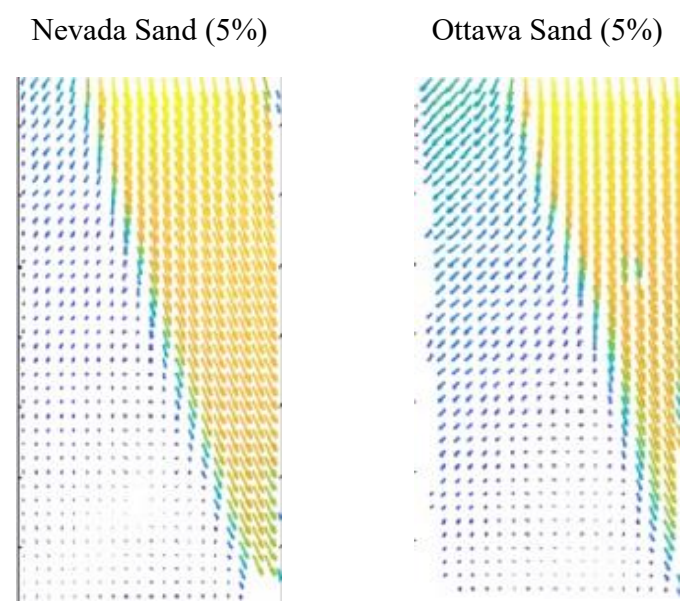

Fig. 6. Vector analysis of particle movement

\section{Conclusion}

The movement of soil particles was observed using Particle Image Velocimetry (PIV) method. The main advantage of this PIV, GeoPIV-RG, is that horizontal and vertical movements are able to be considered separately. Also, this analysis method provides not only displacement contour but also displacement vector. Therefore, it was very easy to understand the particle movement during shearing. From several previous researches, it has been known that shear bands start to be formed immediately after peak stress. From the surface, it was true that shear bands could not be observed during shearing stage. However, in the horizontal point of view, it was possible to expect the location and angle of shear band before peak stress though PIV analysis. Therefore, it was easier to understand and estimate shear bands formation using horizontal movement of soil particles than vertical or resultant movement of soil particles. On PIV results, soil particles did not move much during shearing stage and started to move during residual stress state.

\section{References}

1. T. Yoshida, F. Tatsuoka, M.S.A. Siddiquee \& Y. Kamegai, C. S. Park, Localisation and Bifurcation Theory for Soils and Rocks (1994)

2. M. Oda, H. Kazama, Geotechnique, 48, No.3, 465481 (1998)
3. Amy L. Rechenmacher, Richard J. Finno, Geotechnical Testing Journal, Vol. 27, No. 1 (2004)

4. H.B. Mason, N.W. Trombetta, S.M. Gille, J.N. Lund, J.D. Zupan, H. Puangnak, B.Y. Choy, Z. Chen, C. Bolisetti, J.D. Bray, T.C. Hutchinson, G.L. Fiegel, B.L. Kutter, A.S. Whittaker, Seismic performance assessment in dense urban environments: centrifuge data report for HBM03 (2013)

5. A.M.P Bastidas, Ottawa F-65 Sand Characterization (2016)

6. H. I. Ling, F. Tatshuoka, Journal of Geotechnical Engineering, Vol. 120, No. 7 (1994)

7. S.A Stanier, J. Blaber, W.A. Take, D.J. White, Improved image-based deformation measurement for geotechnical applications, Canada Geotechnical Journal, Vol. 53 (2016) 